\title{
Entretien avec Richard Millet
}

Jean-Yves Laurichesse

\section{(2) OpenEdition}

Journals

Édition électronique

URL : https://journals.openedition.org/ccs/487

DOI : $10.4000 /$ ccs.487

ISSN : 2558-782X

\section{Éditeur :}

Presses universitaires de Rennes, Association des lecteurs de Claude Simon

\section{Édition imprimée}

Date de publication : 30 juin 2005

Pagination : 139-147

ISBN : 2914518676

ISSN : $1774-9425$

\section{Référence électronique}

Jean-Yves Laurichesse, «Entretien avec Richard Millet », Cahiers Claude Simon [En ligne], 1 | 2005, mis en ligne le 20 septembre 2017, consulté le 01 mars 2022. URL : http://journals.openedition.org/ccs/ 487 ; DOI : https://doi.org/10.4000/ccs.487 


\section{Entretien avec Richard Millet}

Le nom de Claude Simon revient à plusieurs reprises dans des entretiens ou des textes de vous, dans Le Sentiment de la langue ou, récemment, dans Fenêtre au crépuscule. En même temps, vous appartenez à une génération de romanciers que l'on présente souvent comme ayant tourné la page du Nouveau Roman, avec l'arrière-pensée que ce courant aurait conduit le genre romanesque dans une sorte d'impasse. Pourriez-vous nous éclairer sur les circonstances et l'état d'esprit dans lesquels vous avez abordé cette æuvre et avez continué à la lire?

Non pas une impasse, mais, bien au contraire, une leçon du Nouveau Roman, des ouvertures, et non la pure invention formelle (il y avait eu Proust, Joyce, Faulkner, Roussel, Borges), mais le redéploiement intransigeant de ces inventions. Écrire, c'est trouver des solutions narratives (stylistiques, structurelles) et j'étais, à dix-huit ans, en quête de solutions qui ne soient pas des recettes. J'ai commencé par Le Palace, un peu par hasard, très jeune, en même temps que je lisais Butor et Robbe-Grillet (mais, étrangement, nul autre auteur du Nouveau Roman), frappé par le phrasé, les parenthèses, le participe présent, le rythme lent et implacable d'une phrase si étrangement musicale pour un écrivain du regard et de la mémoire. Et puis, les titres m’ont hanté, magnifiques, irréfutables, et simples, donc classiques : La Route des Flandres, Le Vent, L'Herbe, Les Géorgiques.

À propos de ce phrasé musical, on ne peut quêetre frappé par l'évolu- 
tion qui vous a conduit vous-même, après des premiers livres tels que L’Angélus, La Chambre d'ivoire, L'Écrivain Sirieix, brefs récits aux phrases acérées mais classiques, vers des romans plus amples - à partir de La Gloire des Pythre - dont l'écriture se caractérise par l'expansion rythmique et sémantique de la phrase. Cette évolution doit-elle quelque chose à la lecture de Claude Simon, ou s'est-elle accomplie par de tout autres voies?

Dans quelle mesure nous sommes influencés, marqués, impossible de le dire précisément, même si je peux faire état d'une hantise : le sous-titre du Vent, tentative de restitution d'un retable baroque. Audelà de ce qu'il "signifie ", m'importe le mot restitution, qui pourrait définir mon projet à partir de La Gloire des Pythre, la restitution (celle d'une mémoire, d'un monde perdu, historiquement condamné) ne pouvant que se faire dans une grande forme, et cette forme ne pouvant qu'user d'une phrase elle-même d'une grande ampleur - un déploiement plus baroque que classique, sauf si on se réfere à la définition de Ponge selon qui le classicisme est la corde la plus tendue du baroque. Ce qui m'a frappé chez Simon, car on cherche toujours quelque chose de l'ordre du secret chez un écrivain, c'est son emploi souverainement musical et structurant du participe présent ; mais est-ce bien conscient ? Le savais-je en en usant à mon tour ? De même savais-je que je m'inscrivais dans une filiation (qui reste à établir) rassemblant Saint-Simon, Chateaubriand, Melville, Dostoïevski, Proust, Faulkner, Alejo Carpentier, Lobo Antunes ? Ceci dit, la musique symphonique, notamment la musique post-romantique, a joué, par métaphore autant que par hantise structurelle (harmonie, contrepoint, polyphonie), un rôle considérable dans l'évolution de ma phrase.

"Restitution" ou "tentative de restitution" d'une mémoire? La question traverse toute l'auvre de Claude Simon, qui fascine par son pouvoir de représentation tout en mettant constamment en question la possibilité même de représenter quoi que ce soit de "réel " par le moyen des mots, avec le retour lancinant des "comment savoir?" ou "comment était-ce?". Ce doute, caractéristique de "l'ère du soupçon", est-il encore présent dans votre entreprise de restitution du "monde perdu " des paysans de la haute Corrèze, ou bien le roman a-t-il retrouvé selon vous, à la fin du $X X^{e}$ siècle, une certaine assurance dans son rapport au réel? 
Est-ce que la dimension de l'epos, à l'œuvre chez Simon, au moins dans le ton, n'est pas une sorte de pacte (la "tentative ") avec la représentation : une mise en question de la représentation qui garderait ses prestiges au cœur du soupçon, de la déconstruction, de sa mise en scène ? Dans mes romans, la narration est confiée à des personnages qui ont des points de vue, qui doutent, qui se reprennent, qui se contredisent parfois. Cette restitution se fait dans l'incertitude : voyez Le Renard dans le nom, un récit rapporté qui lui-même inscrit dans son corps d'autres récits, et où plusieurs fins sont proposées. On ne peut plus être naïf ; le roman n'a pas plus d'assurance face au réel, aujourd'hui ; d'une certaine façon, il n'y a plus de roman français ; ce qu'on appelle ainsi est pris dans une logique commerciale qui cherche à diversifier ses produits : inflation de sous-produits copiant des modèles (américains, latino-américains). On est dans une post-modernité qui a la nostalgie de modèles anciens, qui tend à travestir ses entrechats sur le vide en regard sociologique. Le roman français est une fiction en quête de ses propres auteurs.

Votre ouvre, et quelques autres, tendraient cependant à prouver qu'il ne faut pas désespérer, et ce que vous dites de votre rapport à Claude Simon, et en amont à Dostoïevski, Proust ou Faulkner, montre bien que l'histoire du roman continue, que des passages de témoins s'opèrent toujours. En ce qui concerne cette "tentative de restitution "d'un monde rural dont vous dites avoir connu la fin à partir des années 60, le thème de la terre tel qu'il traverse l'ceuvre de Claude Simon, dans Les Géorgiques bien sûr, mais aussi dans L'Herbe ou dans L'Acacia, a-t-il pour vous une résonance particulière? Vous a-t-il encouragé à évoquer cet univers dans une autre dimension que celle du roman de terroir, à travers "une parole exigeante ", pour emprunter une expression de Ludovic Janvier à propos du Nouveau Roman?

Du moins est-ce à partir de ce désespoir que j'écris, aujourd'hui, de la même façon que j'ai écrit, il y a une douzaine d'années, à partir de la mort du monde rural ; un monde très peu "tendance ", aujourd'hui, comme disent les imbéciles, malgré Faulkner, Giono (mal perçu, celui-là, encore suspect de pagnolades lyrico-pétainistes, alors qu'il est prodigieusement inventif), dans une moindre mesure 
les récits de Trassard, et bien sûr les romans de Simon que vous citez, qui ont donné, par leur appartenance au Nouveau Roman, une légitimité - laquelle reste d'ailleurs à démontrer constamment, par un écart à maintenir entre la nostalgie d'un monde perdu et la nécessité de dire, avec acharnement, ce que ce monde fut, dans une modernité paradoxale puisque ce n'est pas là, je le répète, une thématique très « française ".

Lorsque Claude Simon, dans L'Acacia, évoque sa lignée paternelle, son grand-père paysan pauvre du Jura, ses tantes institutrices de campagne, il est en effet loin de toute nostalgie, insistant sur la dureté de la vie matérielle, l'étroitesse de l'horizon intellectuel. Et pourtant ces pages, portées par la grande phrase simonienne, rendent hommage à ces humbles, conférant à leurs vies une sorte de gloire qui est celle de la littérature. Cela rejoint l'epos dont vous parliez. On retrouve chez vous, à propos des paysans du plateau de Millevaches, ce phénomène d'intensification, d'amplification par l'écriture, étendu à des romans entiers. S'il ne peut y avoir de nostalgie de ce "monde perdu ", il ne s'agit pas pour autant d'un simple témoignage...

Ce qui est perdu, dès lors qu'on s'y mesure par l'écriture, ne constitue-t-il pas avant tout un formidable appel d'air qu'il s'agit de tordre, de tresser, de ployer en un phrasé qui est à la fois hommage, témoignage, déploration et refus de toute nostalgie, tout en étant une manière d'atteindre à la vérité de ce qui fut ?

Ce passé a aussi chez vous, comme chez Claude Simon et bien sûr chez Proust, une dimension familiale, autobiographique. Claude Simon, tout en conservant la référence générique au roman, déclare volontiers qu'il y a de moins en moins de fiction dans ses livres. De votre côté, si la dimension personnelle semble s'affirmer davantage encore dans votre dernier roman, Ma vie parmi les ombres, vous restez attaché à la notion de fiction, de transposition romanesque. Vous reconnaissez-vous dans ce quion a pu appeler un "retour à la fiction", ou bien privilégiez-vous cette écriture de soi que le prière d'insérer du Jardin des Plantes définit comme "portrait d'une mémoire"?

J'ai toujours eu en horreur le "retour à » : retour à la terre, à Bach (Stravinsky), à la fiction. Le roman est pour moi un lieu d'oscillation entre le romanesque et le mémoriel, dans l'indécidable, l'irre- 
pérable, l'impossible départ entre fiction et réalité, entre transposition et obsession du détail révélateur, du perdu, du jadis ; le roman est avant tout pour moi un rythme de langue ; c'est pourquoi j'ai pu dire que Les Mémoires d'Outre-Tombe, Le Soulier de satin, Amers, les Antimémoires sont à mettre au compte des grands romans français.

Vous partagez donc le point de vue de Claude Simon rétorquant à ceux qui sétonnent du sous-titre "roman " attribué à des livres comme L'Acacia ou Le Jardin des Plantes, qu'ils doivent d'abord définir euxmême ce qu'ils entendent par ce mot. En tant que lecteur et en tant qu'écrivain, vous sentez-vous proche de la manière dont Claude Simon élabore, de livre en livre, ce qu'un critique a appelé un "imaginaire biographique ", par l'évocation de ses ascendants et de certains moments de sa propre existence?

Oui, de plus en plus proche, par proustisme (si j'ose dire), Le Tramway étant d'ailleurs placé sous l'égide de Conrad et de Proust, $\mathrm{du}$ " pur » roman et du roman travaillé par l'essai, ou de l'essai romanesque, vous voyez, le genre se cherche - et c'est là, me semble-t-il, que se joue l'avenir du roman : dans sa capacité à se régénérer dans ce qui passait jusque-là pour ses marges. Pour moi, j’ai beau avoir sous-titré roman Ma vie parmi les ombres, la presse a accueilli ce livre comme une autobiographie plus ou moins imaginaire, jouant le réel contre des effets de réel, puisque mon enfance libanaise était gommée de ces recensions, de ces restitutions de ma vie. Ceci dit, tout épisode lointain ne peut apparaître que comme romanesque (ainsi ceux relatés dans Le Tramway évoquent une civilisation disparue qui, en tant que telle, chue dans un temps révolu, est fatalement romanesque, dès lors qu'examinée à partir d'une singularité scripturaire). Idem pour la civilisation rurale dont je suis issu, mais aussi pour la France des années soixante.

Dans Ma vie parmi les ombres, mais déjà dans des romans antérieurs, votre écriture se nourrit d'archives, en particulier photographiques, que le narrateur scrute pour tenter d'atteindre une vérité des êtres disparus. C'est là encore un point de rencontre avec Claude Simon, et j'ai même supposé qu'il pouvait y avoir comme un "clin d'ceil " dans cette phrase de La Gloire des Pythre qui, pour tout 
lecteur de Claude Simon, évoque les cartes postales envoyées par le père dans Histoire : "[...] à moins [...] qu'il ait consenti pour une fois à ne point mettre au feu sans les lire ces cartes postales postées à FortArchambault, Tananarive ou Sä̈gon, sans autre écriture que l'adresse, le seul nom de Pythre et, pour illustration, une indigène aux seins nus ou une scène de genre : marché villageois, buffles, danse sacrée, train de brousse, porteuses d'eau, ou un transatlantique à l'ancre [...]" (Folio, p. 255).

Non, pas de clin d'œil, ni même de souvenir d'Histoire en cours d'écriture, mais, commun à beaucoup de Français, l'arrière-fond colonial déjà légendaire dans mon enfance limousine et que mon autre enfance, au Liban celle-là, ravivait de façon singulière, ces cartes postales, ces noms, ces cartes géographiques où l'empire était figuré en violet nourrissant mon imagination, redoublant les lectures enfantines de Jules Verne, de Joseph Peyré, de Pierre Benoît, mais surtout marquant l'écart entre l'arriération du monde de Siom et le vaste monde, comme disaient ces romans. Si j'ai la passion de l'archive, c'est moins pour donner de l'aplomb à ce que je raconte, que pour sauver l'archive elle-même - notamment ces collections de cartes postales éditées par ma grand-mère et mes deux grandstantes : objets fragiles, que je suis seul à pouvoir commenter, rêvant même d'écrire un livre à partir d'eux...

L'archive est donc aussi pour vous un stimulant (pour ne pas dire un "générateur") de l'écriture. Mais son intégration pose un certain nombre de problèmes formels, surtout si elle est utilisée largement. Avez-vous le sentiment que les solutions inventées par Claude Simon peuvent être utiles à un écrivain d'aujourd'hui (sans qu'il s'agisse bien entendu de les reproduire littéralement), ou sont-elles trop liées à une poétique, celle du Nouveau Roman, qui appartient déjà à l'histoire de la littérature?

Les solutions formelles sont sans doute ce qui, historiquement (et à proportion de leur vertu novatrice) vieillit le plus vite ; Claude Simon n'y échappe pas plus que Butor ou Robbe-Grillet ; et il y a, déjà, une vieillerie esthétique du Nouveau Roman. C'est pourquoi, par exemple, les découpages du début du Jardin des Plantes ou le montage temporel de L'Acacia (qui fait d'ailleurs songer à celui du Bruit et la fureur de Faulkner) me semblent moins intéressants, moins libéra- 
teurs d'énergie fécondante que l'étonnante fluidité narrative du Tramway. Pour moi, j'y reviens, c'est dans le rythme que Simon est le plus novateur. On ne prête pas assez attention à ce qu'une construction peut avoir de musical, donc de hantant. Il y a les écrivains qui hantent, musicalement obsédants, et les autres, qui sont des visuels et, pour moi, sans grand intérêt ; Claude Simon fait partie des premiers, comme Thomas Bernhard ou Antonio Lobo Antunes.

L'archive ne renvoie pas seulement à l'histoire privée des hommes, mais aussi, indissociablement, à leur histoire collective. Elle est présente dans le travail de mémoire que vous avez entrepris, avec en particulier les guerres: 1870, 1914-1918, 1939-1945, guerres coloniales. Cependant, vous appartenez, à la différence de Giono ou de Claude Simon, qui avaient vécu ces épreuves, à la première génération d'écrivains pour qui la guerre relève d'une mémoire indirecte. Il y a donc à la fois continuité et rupture. Comment cela se traduit-il dans votre travail d'écrivain?

Les guerres européennes étaient l'objet de récits permanents, de témoignages directs (notamment la Grande Guerre, qui continue de me fasciner, n'ayant personne qui, dans ma famille, ait fait celle de 39-45); et puis il y avait les gestes coloniales, l'Indochine, surtout. Et puis, pour moi, à Beyrouth, la Guerre des Six Jours, en direct. Ce sont ces récits dont je tente de retrouver aujourd'hui la teneur, l'oralité, le légendaire, la précision. Il y a chez moi, étrangement, comme une nostalgie de ces guerres auxquelles je n'ai pas pris part : déclaration ambiguë, mais qui explique le désir que j’ai d'écrire sur la guerre. Mon premier livre, L'Invention du corps de saint Marc, a pour décor la guerre civile libanaise...

Cette dimension légendaire qui tient, outre les rapports bien connus entre la guerre et le sacré, à la distance temporelle et aux récits intermédiaires, on en trouve quelque chose chez Claude Simon avec l'évocation des guerres de l'Empire dans Les Géorgiques, autour de la figure du Général L.S.M., ou même de la Grande Guerre dans L’Acacia, autour de la figure du père, même si la légende est en même temps mise à distance critique. Mais il y a aussi la manière dont, dans La Route des Flandres ou dans les romans que je viens de citer, sont évoqués les combats vécus de mai 1940, sous une forme violemment émotionnelle, sensorielle, éclatée, dans une tentative, au contraire, 
d'abolir la distance? Etes-vous également sensible, lorsque vous lisez Claude Simon, à ces deux dimensions de la guerre et de sa représentation?

D'autant plus sensible que la question de la guerre est à peu près absente du roman français, qui, avec Balzac et Stendhal, dans Le Colonel Chabert et La Chartreuse, en avait pourtant donné deux versions possibles par le biais de l'intime, de la sphère privée, loin de l'épique de Chateaubriand dans Les Mémoires d'Outre-Tombe. Il est vrai que nous n'avons pas à notre disposition la prodigieuse geste napoléonienne, gigantesque jusque dans sa défaite, et que la débâcle de 1940 offre peu d'images, sinon un destin de téléfilm. C'est pourquoi je sais gré à Claude Simon d'avoir, à ce sujet, ancré en moi quelques scènes mémorables, parce que se rattachant à l'expérience personnelle autant qu'à ce qu'il appelle "les profondeurs de l'Histoire ". J'ai toujours pensé que le destin du roman est lié à la guerre, du moins que le romancier devrait avoir connu la guerre, de près ou de loin, pour mettre à l'épreuve son art poétique. Voyez les Américains de la Génération perdue...

Une dimension importante de la poétique de la guerre chez Claude Simon est d'ordre sensoriel : la guerre est d'abord quelque chose qui arrive au corps au-delà des limites ordinaires de la sensation. Mais cette dimension se retrouve partout dans son ouvre, qu'il s'agisse de l'enfance, des voyages, etc. Je suppose qu'elle vous touche particulièrement, puisque le sensoriel joue un rôle très important dans vos livres ?

Oui, la musicalité du sensoriel, quelque chose de rare dans les œuvres contemporaines. Si je me réfere à la Guerre des Six jours, telle que je l'ai vécue à Beyrouth, elle est inséparable des odeurs de la ville, et m'en donne même une odeur : celle de la guerre, hallucinatoire et très réelle en même temps, en un jeu de synesthésies très puissantes chez moi, qui n'ai par exemple jamais séparé la couleur de son double sonore ni de ses métamorphoses odoriférantes. Beyrouth, Siom, sont pour moi des mondes d'odeurs sonores, bien plus que visuels. Et ce n'est pas seulement lié à l'enfance ; les synesthésies ne sont pas qu'une forme de nostalgie non psychologique ; elles m'accompagnent en permanence... 
Pour conclure cet entretien, pourriez-vous évoquer Claude Simon à travers un souvenir de lecture qui se serait plus particulièrement imprimé dans votre mémoire?

Un souvenir, ou plutôt une image récurrente, venue d'assez loin : celle de l'écrivain socialement en marge, partageant son temps entre Paris et sa terre d'origine ; une image à la fois réelle et surinvestie par ma lecture en cours, celle des Géorgiques, à la sortie du livre. La figure de l'écrivain telle qu'elle entre dans l'imaginaire du lecteur, instaurant une strate singulière de la lecture, faisant partie de l'histoire de sa lecture.

Réponses écrites à des questions posées par Jean-Yves Laurichesse, février-mars 2005 